\title{
Successful Treatment of Methimazole-Induced Severe Aplastic Anemia by Granulocyte Colony-Stimulating Factor, Methylprednisolone, and Cyclosporin
}

\author{
Munehiro Honda ${ }^{1,2}$ \\ ${ }^{1}$ Fourth Department of Medicine, Teikyo University School of Medicine, Kawasaki, Japan \\ ${ }^{2}$ Department of Endocrinology and Metabolism, Mishuku Hospital, 5-33-12 Kamimeguro, Meguro-ku, Tokyo 153-0051, Japan
}

Correspondence should be addressed to Munehiro Honda, mhonda-endo@umin.ac.jp

Received 17 January 2011; Accepted 24 February 2011

Academic Editors: A. De Bellis and O. Nilsson

Copyright () 2011 Munehiro Honda. This is an open access article distributed under the Creative Commons Attribution License, which permits unrestricted use, distribution, and reproduction in any medium, provided the original work is properly cited.

A 52-year-old Japanese woman was examined because of general malaise, weight loss and a lump in her left breast. She was diagnosed with cancer of the left breast and Graves' disease, and was administered methimazole (MMI). A left mastectomy was performed for the breast cancer. She presented with a high fever and peripheral blood examination revealed a severe pancytopenia. She was diagnosed with severe aplastic anemia, and administered G-CSF, however, the treatment was unsuccessful. Thus, oral methyprednisolone and cyclosporin were added. There was a remarkable improvement in the peripheral blood count.

\section{Introduction}

Methimazole (MMI) is widely used to treat Graves' disease. Agranulocytosis is a severe side effect and its frequency is reported to be $0.18-0.55 \%$ [1-5]. Aplastic anemia (AA) is very rare in MMI treatment and most cases rapidly recover after cessation of MMI therapy [6-13]. Granulocyte colonystimulating factor (G-CSF), glucocorticoid and cyclosporin have only been used in severe cases. I report here a case of MMI-induced severe AA associated with severe bacterial infection. The patient was treated with G-CSF after cessation of MMI, but her peripheral blood count did not recover even when she was administered a higher dosage of GCSF for 11 days. Therefore, we administered a combination of methyprednisolone and cyclosporin and, her peripheral blood count and bone marrow rapidly recovered.

\section{Case Report}

A 52-year-old Japanese woman was examined in March, 2003 because of general malaise, weight loss and a lump in her left breast. She was diagnosed with cancer of the left breast and Graves' disease, and was administered MMI $30 \mathrm{mg} /$ day for Graves' disease. Her thyroid function was controlled with
MMI, and a left mastectomy was performed for breast cancer one month later. She presented with a sudden fever of $39^{\circ} \mathrm{C}$ and a sore throat 12 days after the operation. The peripheral blood examination revealed a white blood-cell count of $0.8 \times 10^{9} / \mathrm{L}$, with $12 \%$ neutrophils, $3 \%$ monocytes, $2 \%$ eosinophils, $83 \%$ lymphocytes, hemoglobin concentration of $9.5 \mathrm{~g} / \mathrm{dL}$, and platelet count of $4 \times 10^{9} / \mathrm{L}$; therefore, MMI therapy was stopped and she was administered $50 \mu \mathrm{g}$ of GCSF subcutaneous injection and $2.5 \mathrm{~g}$ of gamma globulin intravenous infusion. The treatment was unsuccessful, and she was transferred to our hospital because of progressive pancytopenia and a constant high fever for a week. On admission, she complained of a sore throat, chills and general malaise. A physical examination revealed a high fever $\left(39^{\circ} \mathrm{C}\right)$, sinus tachycardia, grade II systolic murmur, exophthalmos, moderate diffuse goiter and gingival bleeding. The palpebral conjunctiva was anemic. No superficial lymph nodes were swollen. A Penrose drain was left in her left breast, and exudates were noted. Oropharyngeal examination revealed tonsillitis without white curd-like plaque. Electrocardiogram showed sinus tachycardia. Chest X-ray was normal. Peripheral blood examination showed a white blood-cell count of $0.8 \times 10^{9} / \mathrm{L}$, with $2 \%$ neutrophils, hemoglobin concentration of $8.5 \mathrm{~g} / \mathrm{dL}$, platelet count of $3 \times 10^{9} / \mathrm{L}$, and reticulation of red 
blood cells of $1.3 \%$. Laboratory examinations showed normal liver function and renal function. Serum albumin was $3.0 \mathrm{~g} / \mathrm{dL}$, alkaline phosphatase was $260 \mathrm{IU} / \mathrm{L}$, total cholesterol was $92 \mathrm{mg} / \mathrm{dL}$, and C-reactive protein (CRP) was $16.9 \mathrm{mg} / \mathrm{dL}$. Throat and blood bacterial cultures were negative, but exudate culture from her left breast yielded Staphylococcus species. Thyroid function tests were TSH $<0.002 \mathrm{mIU} / \mathrm{L}$, free triiodothyronine (FT3) $>20.0 \mathrm{pg} / \mathrm{mL}$, free thyroxine (FT4) $>8.0 \mathrm{ng} / \mathrm{dL}$, and $\mathrm{TSH}$ receptor antibody $19.7 \mathrm{IU} / \mathrm{L}$; thus MMI-induced severe AA was strongly suspected, and bone marrow (BM) aspiration was performed immediately. There were decreased nucleated cells with fat replacement in BM (Figure 1(a)). The patient was administered iodide $126 \mathrm{mg} /$ day and imipenem/cilastatin $2 \mathrm{~g} /$ day and G-CSF was increased to $300 \mu \mathrm{g} /$ day. Two days later, intravenous amikacin (400 mg/day) was added; however, with a progressive depression in peripheral blood count, the patient needed transfusions of erythrocytes and platelets. Five days later, oral methyprednisolone and cyclosporin were added. There was a remarkable improvement of the high fever and pancytopenia, and normalization of the CRP. G-CSF was stopped 8 days later, and methyprednisolone and cyclosporin were stopped 10 days later. A second bone marrow aspiration was performed 3 weeks later and BM was already normocellular (Figure 1(b)); thus she was administered ${ }^{131} \mathrm{I}$ radioniodine, and 2 months later her thyroid function was normal. The clinical course of this case is illustrated in Figure 2.

\section{Discussion}

AA is very rare complication as a result of the administration of MMI [6-13]. The pathogenic mechanisms of agranulocytosis and AA as a result of the administration of MMI are unclear; however, direct cytotoxic effect by MMI or autoimmune reaction for bone marrow precursors are candidates of pathogenic mechanisms. Previously, it was reported that agranulocytosis was developed in patients administered more than $40 \mathrm{mg}$ /day MMI; however, agranulocytosis developed regardless of the dosage and duration of MMI administration [1, 2]. Tamai et al. found that HLA DRB1*08032 related to MMI induced agranulocytosis in Japanese [14]; however, our patient did not have it. Weitzman and Stossel reported expression of antineutrophil antibody belonging to IgM in the sera of patients who developed MMI induced agranulocytosis [15]. It is reported that incubation with autologous sera of a patient with MMI induced AA collected on earlier days resulted in a reduced number of colony-forming units in culture in peripheral blood mononuclear cells, and autoimmune humoral reactions may occur against myeloid precursors [7]. In addition, MMI induces other autoimmune disorders such as hemolytic anemia, insulin autoimmune syndrome and lupus-like syndrome [16-18]. Thus, the intervention of autoimmune abnormality may be more causal than the cytotoxic effect.

Most cases of MMI induced AA usually develop within 3 months after the start of therapy [6]. In our patient, bone marrow aplasia occurred about 1.5 months after the start

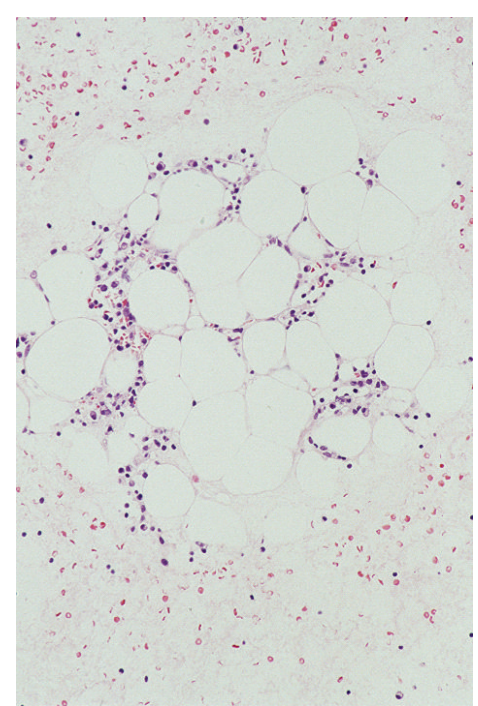

(a)

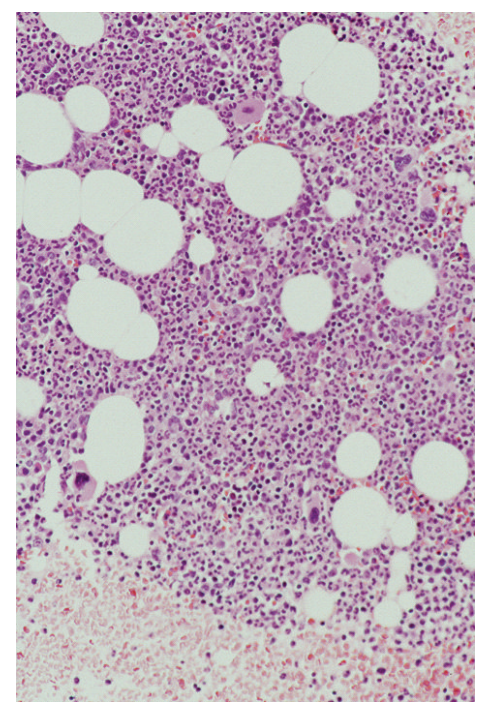

(b)

FIgure 1: Photomicrographs of bone marrow aspiration. (a) Decreased nucleated cells with fat replacement in bone marrow at diagnosis (b) Increased nucleated cells with an absence of fat replacement (HE staining $\times 100$ ).

of MMI. Moreover, recovery is usually prompt within 25 weeks from the withdrawal of MMI and the start of GCSF, and bone marrow transplantation and antithymocyte globulin are seldom needed [6]. However, the withdrawal of MMI and the administration of G-CSF for 11 days had little effect on the peripheral blood count in the patient. Therefore, oral methylprednisolone and cyclosporin were added, the neutrophils count rapidly increased and the erythrocyte and platelet counts gradually increased. Escobar-Morreale et al. reported that in the case of MMI induced AA patient who had no response with granulocyte-monocyte colonystimulating factor for a week, standard immunosuppressive drugs should be susbstituted [8]. As the course of MMI induced AA is highly variable, it is not known whether bone 


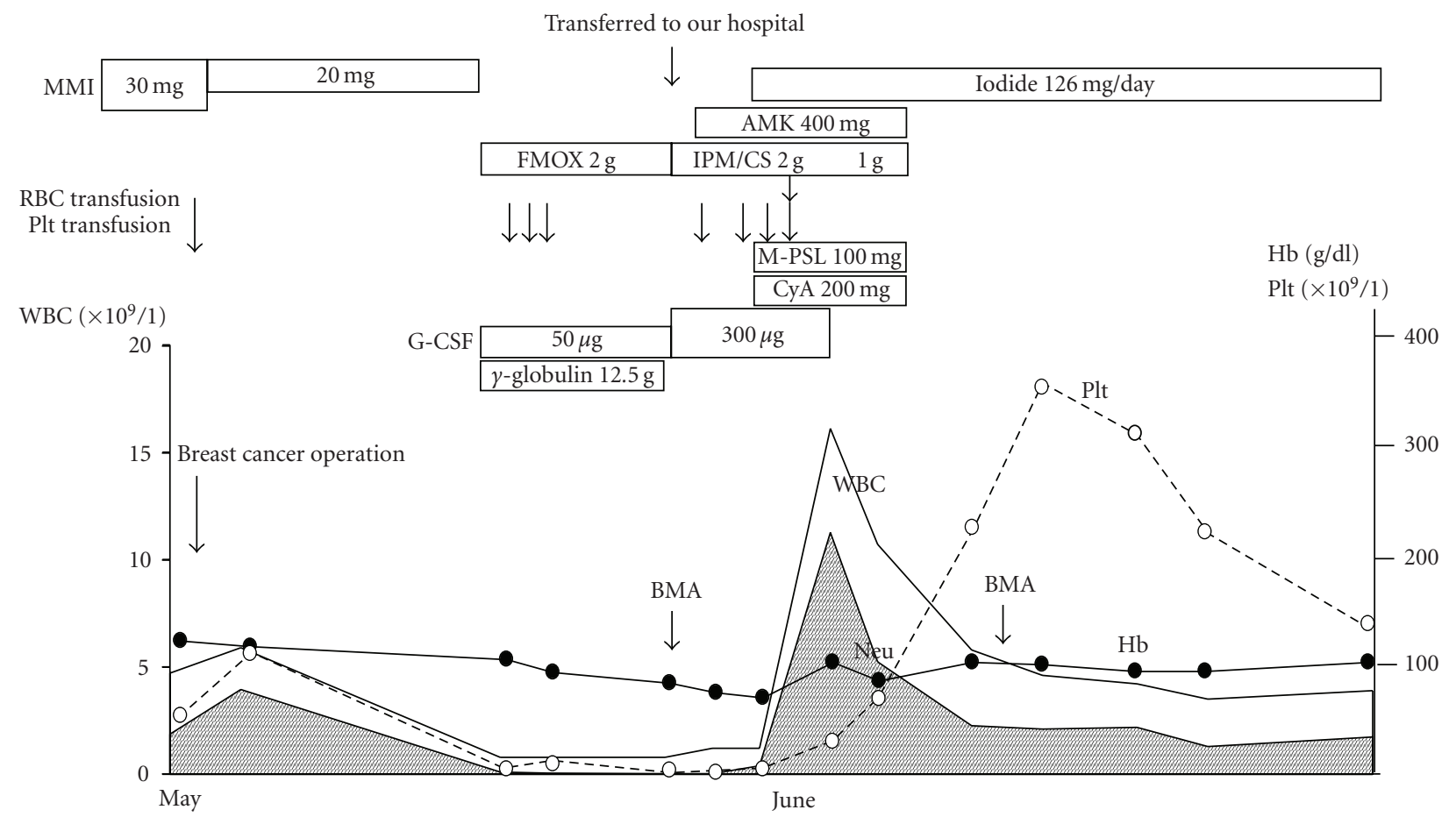

FIGURE 2: Illustration of the clinical course of the case. MMI: methimazole, FMOX: flomoxef, AMK: amikacin, IPM/CS: imipenem/cilastatin, M-PSL: methyprednisolone, CyA: cyclosporin, BMA: bone marrow aspiration, Neu: neutrophils.

marrow aplasia recovered spontaneously or whether recovery was a result of the administration of G-CSF or methylprednisolone and cyclosporin. It was reported that the recovery of MMI induced agranulocytosis was shortened significantly by the administration of G-CSF and glucocorticoid [19]. However, the administration of G-CSF for 11 days had little effect on the peripheral blood count in our patient. It could not be determined which drug was most effective, but these combinations seemed useful in our patient.

As the patient developed AA with breast cancer two weeks postoperatively, the Penrose drain was left in her left breast, and exudates were noted. Staphylococcus species was detected from the culture of exudates. Immunosuppressive agents such as glucocorticoid and cyclosporin generally aggravate an infectious disease, resulting in restorative delay. For example, our patient suffered from severe bacterial infection; however, bone marrow rapidly recovered after the immunosuppressive agent combination was administered and the severe infection was resolved immediately after administration of antibiotics. This suggests that bone marrow is rapidly recovered by a combination of G-CSF and an immunosuppressive agent under the appropriate administration of antibiotics in a severe AA patient with severe infection.

In summary, I report the successful treatment of MMIinduced severe AA by G-CSF, methyprednisolone, and cyclosporin. Although AA is a very rare but severe complication of Basedow's disease with MMI treatment, early recovery can be expected as a result of the administration of a combination of G-CSF and immunosuppressive therapy.

\section{References}

[1] D. S. Cooper, "Antithyroid drugs," New England Journal of Medicine, vol. 311, no. 21, pp. 1353-1362, 1984.

[2] D. Reinwein, G. Benker, J. H. Lazarus, and W. D. Alexander, "A prospective randomized trial of antithyroid drug dose in Graves' disease therapy," Journal of Clinical Endocrinology and Metabolism, vol. 76, no. 6, pp. 1516-1521, 1994.

[3] M. Meyer-Gessner, G. Benker, S. Lederbogen, T. Olbricht, and D. Reinwein, "Antithyroid drug-induced agranulocytosis: clinical experience with ten patients treated at one institution and review of the literature," Journal of Endocrinological Investigation, vol. 17, no. 1, pp. 29-36, 1994.

[4] J. Tajiri, S. Noguchi, T. Murakami, and N. Murakami, "Antithyroid drug-induced agranulocytosis: the usefulness of routine white blood cell count monitoring," Archives of Internal Medicine, vol. 150, no. 3, pp. 621-624, 1990.

[5] H. Tamai, Y. Takaichi, T. Morita et al., "Methimazole-induced agranulocytosis in Japanese patients with Graves' disease," Clinical Endocrinology, vol. 30, no. 5, pp. 525-530, 1989.

[6] N. Biswas, Y. H. Ahn, J. M. Goldman, and J. M. Schwartz, "Case report: aplastic anemia associated with antithyroid drugs," American Journal of the Medical Sciences, vol. 301, no. 3, pp. 190-194, 1991.

[7] J. Moreb, O. Shemesh, S. Shilo, C. Manor, and C. Hershko, "Transient methimazole-induced bone marrow aplasia: in vitro evidence for a humoral mechanism of bone marrow suppression," Acta Haematologica, vol. 69, no. 2, pp. 127-131, 1983.

[8] H. F. Escobar-Morreale, P. Bravo, R. García-Robles, J. García-Laraña, H. De La Galle, and J. M. Sancho, "Methimazole-induced severe aplastic anemia: unsuccessful 
treatment with recombinant human granulocyte-monocyte colony-stimulating factor," Thyroid, vol. 7, no. 1, pp. 67-70, 1997.

[9] X. Lopez-Karpovitch, A. Ulloa-Aguirre, C. Von Eiff, R. Hurtado-Munroy, and A. Alanis, "Treatment of methimazole induced severe aplastic anemia with recombinant human granulocyte-monocyte colony-stimulating factor and glucocorticosteroids," Acta Haematologica, vol. 87, no. 3, pp. 148 150, 1992.

[10] P. Mezquita, V. Luna, M. Muñoz-Torres et al., "Methimazoleinduced aplastic anemia in third exposure: successful treatment with recombinant human granulocyte colonystimulating factor," Thyroid, vol. 8, no. 9, pp. 791-794, 1998.

[11] A. Yamamoto, Y. Katayama, K. Tomiyama et al., "Methimazole-induced aplastic anemia caused by hypocellular bone marrow with plasmacytosis," Thyroid, vol. 14, no. 3, pp. 231235, 2004.

[12] J. Jakucs and G. Pocsay, "Successful treatment of methimazoleinduced severe aplastic anemia with recombinant human granulocyte colony-stimulating factor and high-dosage steroids," Journal of Endocrinological Investigation, vol. 29, no. 1, pp. 74-77, 2006.

[13] D. Thomas, A. Moisidis, A. Tsiakalos, K. Alexandraki, V. Syriou, and G. Kaltsas, "Antithyroid drug-induced aplastic anemia," Thyroid, vol. 18, no. 10, pp. 1043-1048, 2008.

[14] H. Tamai, T. Sudo, A. Kimura et al., "Association between the $\mathrm{DRB}^{*} 08032$ histocompatibility antigen and methimazoleinduced agranulocytosis in Japanese patients with Graves disease," Annals of Internal Medicine, vol. 124, no. 5, pp. 490494, 1996.

[15] S. A. Weitzman and T. P. Stossel, "Drug-induced immunological neutropenia,” Lancet, vol. 1, no. 8073, pp. 1068-1072, 1978.

[16] A. Salama, H. Northoff, H. Burkhardt, and C. MuellerEckhardt, "Carbimazole-induced immune haemolytic anaemia: role of drug-red blood cell complexes for immunization," British Journal of Haematology, vol. 68, no. 4, pp. 479-482, 1988.

[17] Y. Hirata, M. Tominaga, J. I. Ito, and A. Noguchi, "Spontaneous hypoglycemia with insulin autoimmunity in Graves' disease," Annals of Internal Medicine, vol. 81, no. 2, pp. 214 218, 1974.

[18] E. M. Berkman, J. B. Orlin, and J. Wolfsdorf, "An antineutrophil antibody associated with a propylthiouracilinduced lupus-like syndrome," Transfusion, vol. 23, no. 2, pp. 135-138, 1983.

[19] H. Tamai, T. Mukuta, S. Matsubayashi et al., "Treatment of methimazole-induced agranulocytosis using recombinant human granulocyte colony-stimulating factor (rhG-CSF)," Journal of Clinical Endocrinology and Metabolism, vol. 77, no. 5, pp. 1356-1360, 1993. 


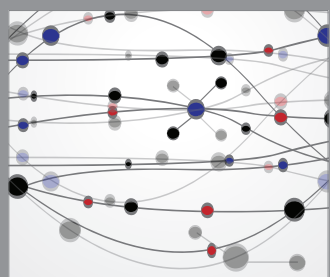

The Scientific World Journal
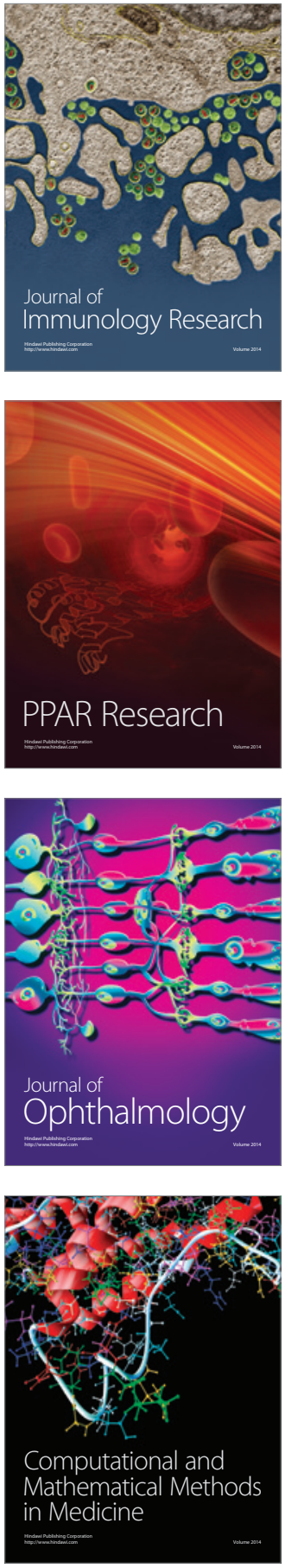

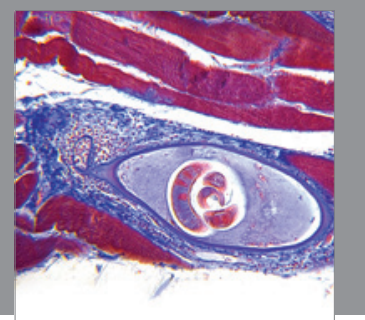

Gastroenterology

Research and Practice
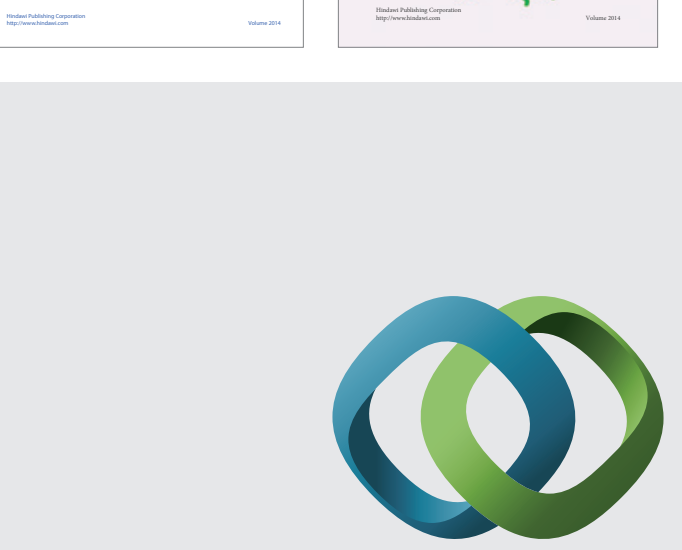

\section{Hindawi}

Submit your manuscripts at

http://www.hindawi.com
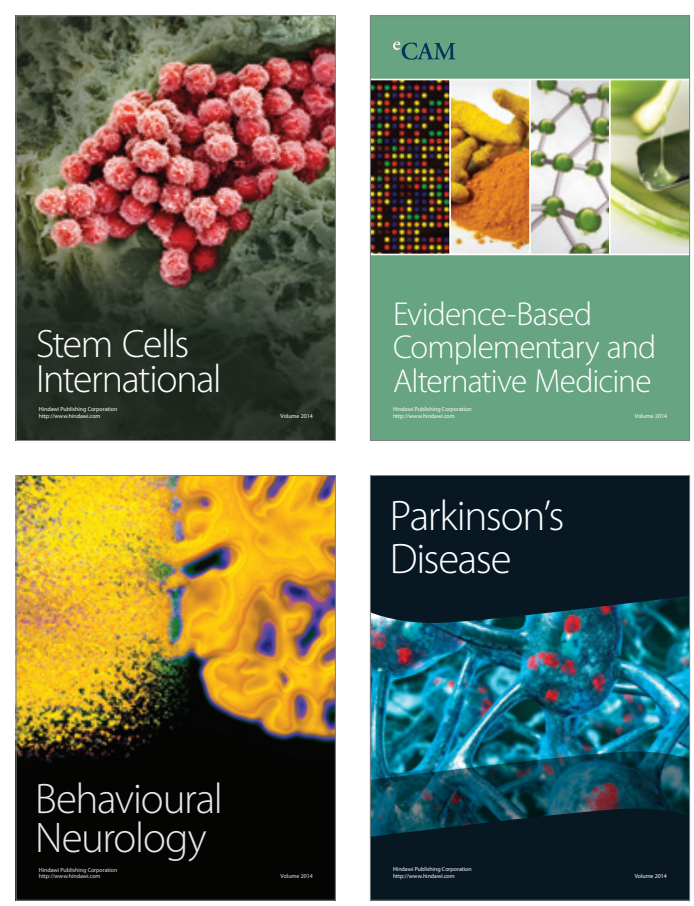

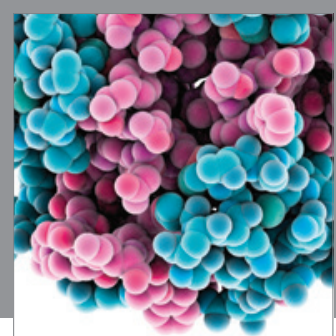

Journal of
Diabetes Research

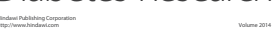

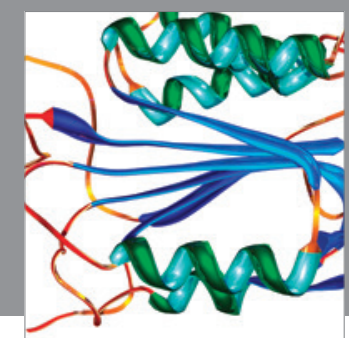

Disease Markers
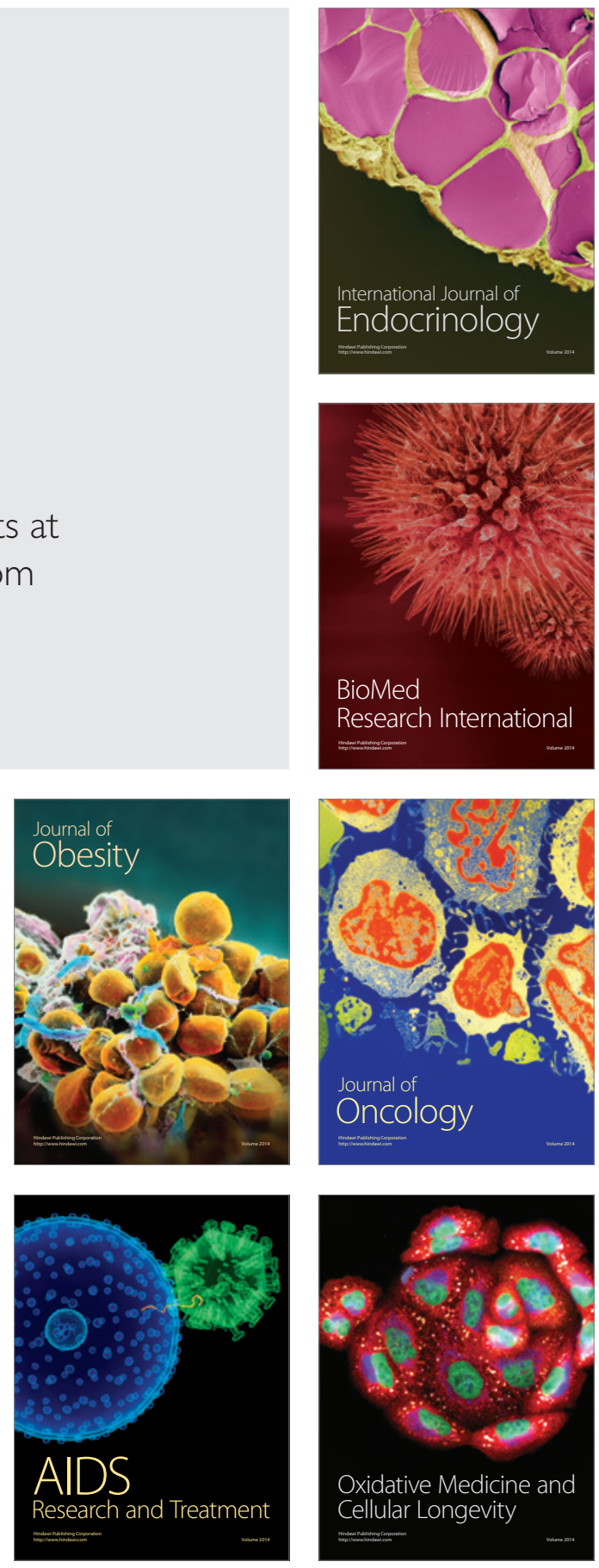